\title{
Mycosubtilin Produced by Bacillus subtilis ATCC6633 Inhibits Growth and Mycotoxin Biosynthesis of Fusarium graminearum and Fusarium verticillioides
}

\author{
Chenjie Yu ${ }^{1,+}{ }^{,}$Xin Liu ${ }^{2,3,+}$, Xinyue Zhang ${ }^{1}$, Mengxuan Zhang ${ }^{1}$, Yiying Gu ${ }^{1}$, Qurban Ali ${ }^{1}$, \\ M. Sherif Ramzy Mohamed ${ }^{4}$, Jianhong Xu ${ }^{2,3}$, Jianrong Shi ${ }^{2,3}$, Xuewen Gao ${ }^{1}$, Huijun $\mathrm{Wu}^{1}$ and Qin Gu ${ }^{1, *}$ \\ 1 Key Laboratory of Integrated Management of Crop Diseases and Pests, Department of Plant Pathology, \\ College of Plant Protection, Nanjing Agricultural University, Nanjing 210095, China; \\ yuchenjie0501@163.com (C.Y.); xyzhang0661@163.com (X.Z.); zhangmengxuan0927@163.com (M.Z.); \\ gyiying520@163.com (Y.G.); qurbanalirattar@webmail.hzau.edu.cn (Q.A.); gaoxw@njau.edu.cn (X.G.); \\ hjwu@njau.edu.cn (H.W.) \\ 2 Institute of Food Safety and Nutrition, Jiangsu Academy of Agricultural Science, Nanjing 210014, China; \\ xinliu@jaas.ac.cn (X.L.); xujianhongnj@126.com (J.X.); jianrong63@126.com (J.S.) \\ 3 School of Food and Biological Engineering, Jiangsu University, Zhenjiang 212013, China \\ 4 Department of Food Toxicology and Contaminant, National Research Centre of Egypt, Giza 12411, Egypt; \\ sheriframzy4@gmail.com \\ * Correspondence: guqin@njau.edu.cn \\ + These authors contributed equally to this work.
}

Citation: Yu, C.; Liu, X.; Zhang, X.; Zhang, M.; Gu, Y.; Ali, Q.; Mohamed, M.S.R.; Xu, J.; Shi, J.; Gao, X.; et al. Mycosubtilin Produced by Bacillus subtilis ATCC6633 Inhibits Growth and Mycotoxin Biosynthesis of Fusarium graminearum and Fusarium verticillioides. Toxins 2021, 13, 791. https://doi.org/10.3390/ toxins13110791

Received: 20 August 2021

Accepted: 1 November 2021

Published: 9 November 2021

Publisher's Note: MDPI stays neutral with regard to jurisdictional claims in published maps and institutional affiliations.

Copyright: (c) 2021 by the authors. Licensee MDPI, Basel, Switzerland. This article is an open access article distributed under the terms and conditions of the Creative Commons Attribution (CC BY) license (https:// creativecommons.org/licenses/by/ $4.0 /)$.
Abstract: Fusarium graminearum and Fusarium verticillioides are fungal pathogens that cause diseases in cereal crops, such as Fusarium head blight (FHB), seedling blight, and stalk rot. They also produce a variety of mycotoxins that reduce crop yields and threaten human and animal health. Several strategies for controlling these diseases have been developed. However, due to a lack of resistant cultivars and the hazards of chemical fungicides, efforts are now focused on the biocontrol of plant diseases, which is a more sustainable and environmentally friendly approach. In the present study, the lipopeptide mycosubtilin purified from Bacillus subtilis ATCC6633 significantly suppressed the growth of F. graminearum PH-1 and F. verticillioides 7600 in vitro. Mycosubtilin caused the destruction and deformation of plasma membranes and cell walls in F. graminearum hyphae. Additionally, mycosubtilin inhibited conidial spore formation and germination of both fungi in a dose-dependent manner. In planta experiments demonstrated the ability of mycosubtilin to control the adverse effects caused by F. graminearum and F. verticillioides on wheat heads and maize kernels, respectively. Mycosubtilin significantly decreased the production of deoxynivalenol (DON) and Bseries fumonisins $\left(\mathrm{FB}_{1}, \mathrm{FB}_{2}\right.$ and $\left.\mathrm{FB}_{3}\right)$ in infected grains, with inhibition rates of $48.92,48.48,52.42$, and $59.44 \%$, respectively. The qRT-PCR analysis showed that mycosubtilin significantly downregulated genes involved in mycotoxin biosynthesis. In conclusion, mycosubtilin produced by $B$. subtilis ATCC6633 was shown to have potential as a biological agent to control plant diseases and Fusarium toxin contamination caused by F. graminearum and F. verticillioides.

Keywords: mycosubtilin; Bacillus subtilis; Fusarium graminearum; Fusarium verticillioides; biocontrol; mycotoxins

Key Contribution: Mycosubtilin isolated from Bacillus subtilis ATCC6633 inhibited the growth of F. graminearum PH-1 and F. verticillioides 7600 in vitro and in vivo. Mycosubtilin can suppress the production of DON and B-series fumonisins (FBs) in infected grains.

\section{Introduction}

Fusarium is a cosmopolitan genus of filamentous ascomycete fungi. Fusarium species are toxin-producing plant pathogens that cause wilts, blights, and rots of various crops [1]. 
Fusarium head blight (FHB) disease caused by Fusarium graminearum affects many cereal crops and causes yield losses and reduction of grain quality [2,3]. Fusarium verticillioides causes seedling blight, stalk rot, and ear rot in maize [4,5]. Fusarium spp. produce a wide range of mycotoxins during disease development, and these are harmful to human and livestock health [6,7]. Trichothecenes and fumonisins are the significant and prominent mycotoxins produced by Fusarium species [8].

Deoxynivalenon (DON) and its acetylated derivatives (i.e., 3-DON, 15-DON) are common type B trichothecenes, mainly produced by F. graminearum $[9,10]$. DON is the most abundant mycotoxin detected in cereals, and high incidences and concentrations of DON have also been reported in feedstuffs [11]. DON is a potent inhibitor of protein synthesis, and it causes emesis, immune deficiency, and teratogenic effects in mammals [12]. Fumonisins, mainly synthesized by F. verticillioides, are the most common mycotoxins found in maize. Fumonisins are highly toxic, and their ingestion is associated with human toxicoses, including esophageal cancer, fetal neural tube defects, and hepatitis [13]. The control of Fusarium disease and mycotoxin contamination is challenging. The development of resistant varieties is time-consuming and labor-intensive. Chemical control is the main approach for preventing Fusarium disease, but the consequences of fungicide use include environmental pollution and the development of resistant fungal pathogens [14]. Control of Fusarium disease and mycotoxin accumulation requires alternative strategies and agents that are effective and environmentally friendly. The use of biological agents has attracted attention owing to their safety and efficacy [15].

Plant growth-promoting rhizobacteria (PGPR), which colonize the rhizosphere or plant root, can promote crop yield and suppress many plant pathogens [16]. Many PGPRbased commercial products are available, including Diegall, Galltrol-A, Zea-Nit, Quantum 4000, and Mycostop [17]. The genus Bacillus includes typical PGPR species and is the most promising genus for the development of biopesticides and biofertilizers. This is largely due to their ability to produce many antagonistic secondary metabolites with different structures. For example, Bacillus subtilis devotes approximately $4-5 \%$ of its genome for the synthesis of antibiotics. It can also produce abundant secondary metabolites with antimicrobial activity [18]. Among the active substances, lipopeptides (LPs), composed of a lipid tail linked to a linear or cyclic oligopeptide [19], were first reported to have a vital role in the biocontrol properties of Bacillus. Due to their structures, LPs from Bacillus have been divided into three families: iturin, fengycin (or pilpastatin), and surfactin [20,21]. Fengycins and iturins have strong in-vitro and in-vivo antagonistic activity toward fungal pathogens, including F. graminearum [14,20], Botrytis cinerea [22], Sclerotinia sclerotiorum [23], and Candida albicans $[24,25]$. The surfactin family contains powerful biosurfactants that kill bacteria by interfering with biological membrane integrity. Surfactins also have antiviral and anti-mycoplasma activities and contribute to biofilm formation and rhizosphere competition [26].

This study explored the mechanism underlying the antifungal activity of mycosubtilin and its effect on mycotoxin production. The results showed that the LP mycosubtilin from B. subtilis ATCC6633 can suppress the growth of F. graminearum and F. verticillioides as well as the biosynthesis of mycotoxins, including DON and fumonisins B1, B2, and B3. In addition, gene expression related to $\mathrm{DON}$ and B-series fumonisins (FBs) biosynthesis could be analyzed to show the negative influence of mycosubtilin on the DON and FB pathway. Therefore, we demonstrated that mycosubtilin has great potential as a biological agent, which can control plant diseases and mycotoxin contamination caused by F. graminearum and F. verticillioides.

\section{Results}

2.1. Mycosubtilin Produced by B. subtilis ATCC6633 Displayed In-Vitro Antagonistic Activity against $F$. graminearum and $F$. verticillioides

B. subtilis ATCC6633 has antifungal activity and produces two LPs, namely mycosubtilin and surfactin [27]. The crude antifungal extract was obtained by Amberlite Resin XAD16N extraction. Three peaks, from 11-15 min, for mycosubtilin were found, and it was 
purified by semipreparative HPLC (Figure 1A). The purified mycosubtilin was identified by LC-MS to confirm the existence of three peaks. There were molecular ion peaks $\left((\mathrm{M}+\mathrm{H})^{+}\right)$ for $\mathrm{C}_{15}-\mathrm{C}_{17}$ mycosubtilin at $m / z 1057.6,1071.6$, and 1085.6, which was consistent with a previous report (Figure 2) [28]. The presence of fatty acid chains of varying lengths within mycosubtilin led to 14-Da differences in the molecular mass between several homologues of mycosubtilin [29]. The purity of mycosubtilin, which was used for further study, was found to be $94.3 \%$ via LC-MS analysis and normalization of peak areas. The antimicrobial assay showed that mycosubtilin had strong and long-lasting antagonistic activity against F. graminearum and F. verticillioides, and the inhibition zone had less than a $10 \%$ decrease after an additional $3 \mathrm{~d}$ of incubation (Figure $1 \mathrm{~B}$ and Figure S1). The inhibitory effects of different concentrations of mycosubtilin against $F$. graminearum were determined. Mycosubtilin exerted antifungal activity in a dose-dependent manner at concentrations of $20-500 \mu \mathrm{g} / \mathrm{mL}$ (Figure S2). These results indicated that mycosubtilin inhibited the in-vitro growth of F. graminearum and F. verticillioides.

A

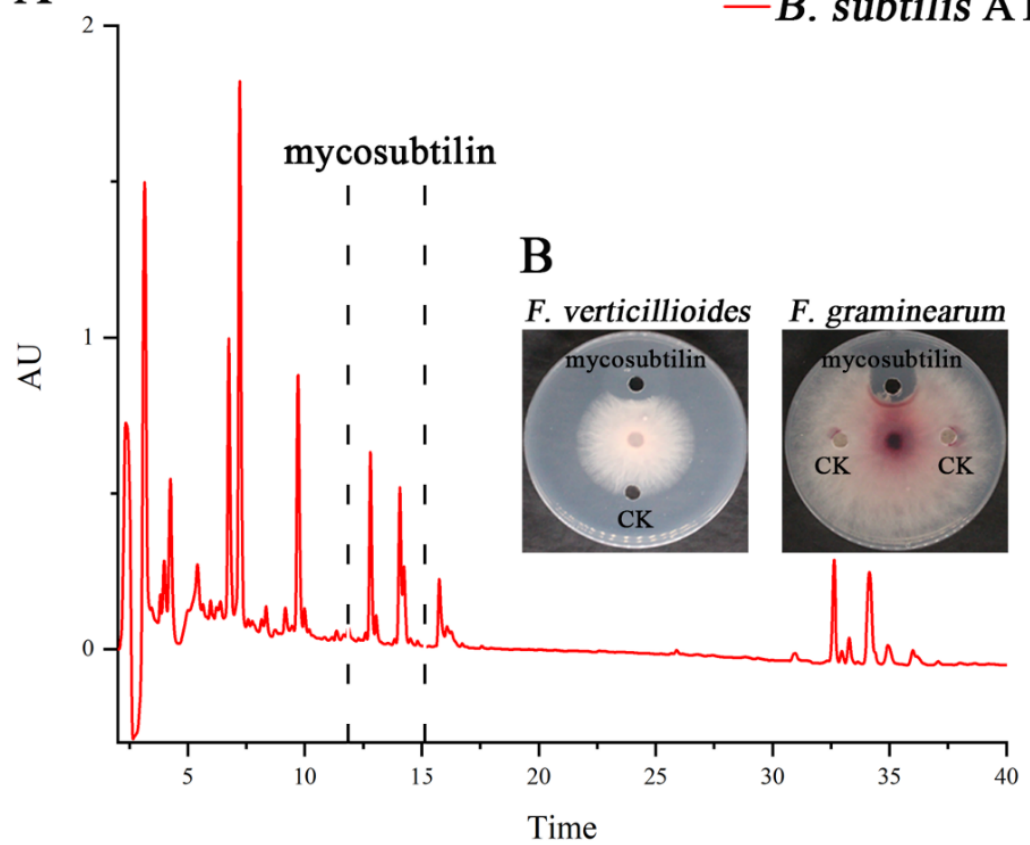

Figure 1. Purification and antifungal assay of mycosubtilin from B. subtilis ATCC6633. (A) Mycosubtilin was isolated from the crude extract of B. subtilis ATCC6633 using semipreparative reverse-phase HPLC. AU (absorbance unit). (B) Assay of antifungal activity using purified mycosubtilin against F. graminearum and F. verticillioides. CK (control), methanol.

\subsection{Ultrastructural Changes in F. graminearum Hyphae Caused by Mycosubtilin}

Transmission electron microscopy (TEM) and scanning electron microscopy (SEM) observations were used to visualize the morphological changes in F. graminearum mycelia under mycosubtilin treatment. The SEM result showed that the untreated control hyphae of F. graminearum were dense, regular, plump, and cylindrical (Figure 3A). In contrast, after being treated with $20 \mu \mathrm{g} / \mathrm{mL}$ of mycosubtilin, destruction and deformation of fungal hyphae were observed, including curling, shrinkage, and breakdown (Figure 3A). Changes in the cellular morphology of $F$. graminearum were also observed using TEM. The untreated fungal hyphae had a well-defined cell wall, intact cell membrane, and uniformly distributed cytoplasm (Figure 3B). Compared with the control, fungal hyphae that had been treated with mycosubtilin showed impaired cellular integrity, irregular structure of the cell wall, and destruction of the cell membrane (Figure 3B). 


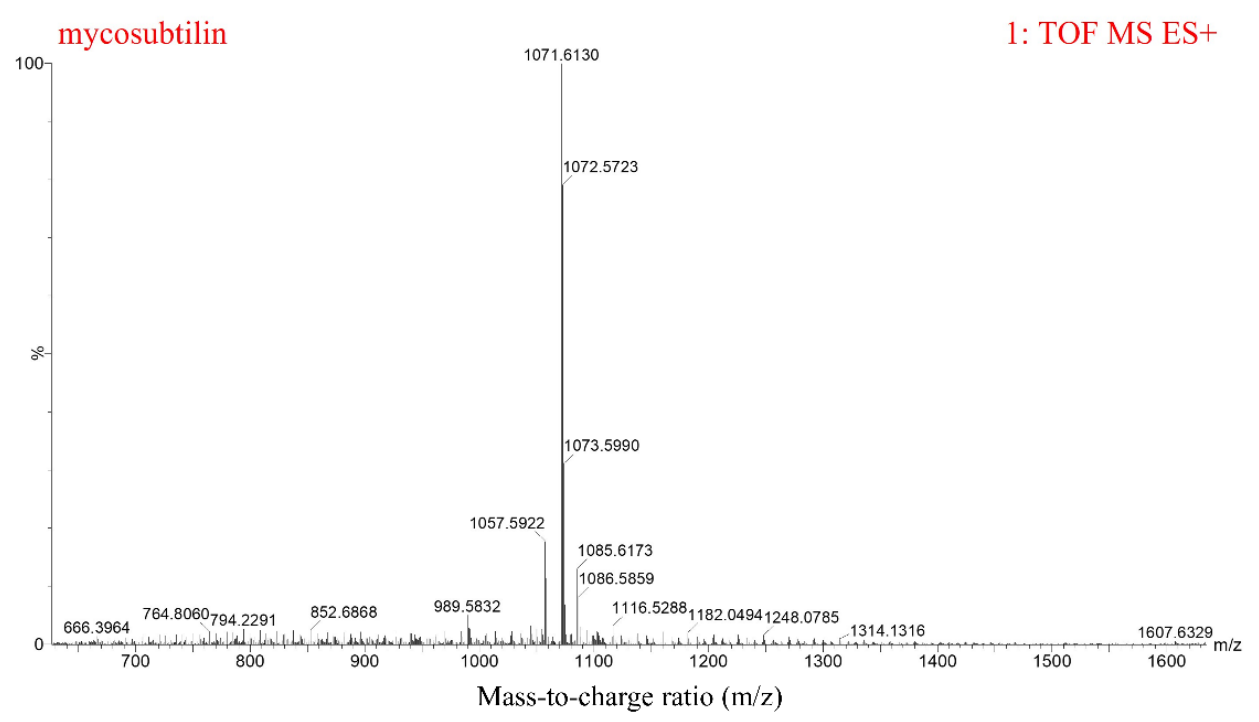

Figure 2. LC-MS analysis of purified mycosubtilin from B. subtilis ATCC6633. The ion peaks at 1057.6, 1071.6, and 1085.6 represent $C_{15}, C_{16}$, and $C_{17}$ mycosubtilin homologues, respectively.

2.3. Mycosubtilin Inhibited the Formation and Germination of Conidial Spores of F. graminearum and $F$. verticillioides

After investigating the hyphal changes induced by mycosubtilin, we studied its effect on fungal spore formation and germination, which are important for F. graminearum and F. verticillioides spread in plants. The formation of fungal spores was suppressed by mycosubtilin in a dose-dependent manner. When mycelia were treated with $20 \mu \mathrm{g} / \mathrm{mL}$ of mycosubtilin, few conidia were observed (Figure 4A,C). The expression analysis of conidiationrelated genes (fgsg_06774,fgsg_02471,fgsg_10166,fveg_05214,fveg_05267,fveg_05331) was conducted to show that mycosubtilin could significantly affect conidiation at the transcriptional level (Figure S3). In the conidial germination assay, different concentrations of mycosubtilin decreased the germination rate of both fungi after treatment of $24 \mathrm{~h}$. After treatment with $50 \mu \mathrm{g} / \mathrm{mL}$ of mycosubtilin for $24 \mathrm{~h}$, the germination rate of F. graminearum and F. verticillioides was only 17.52 and $29.03 \%$, respectively (Figure 4B,D). Additionally, mycosubtilin led to the conidia appearing swollen and dead and inhibited the elongation of germ tubes of both fungi (Figures S4-S6).

\subsection{Mycosubtilin Reduced the Virulence of F. graminearum and F. verticillioides in Plants}

Mycosubtilin had strong in-vitro suppression activity against fungal pathogens. We therefore determined if it could reduce the pathogenicity of F. graminearum and F. verticillioides to plants. Wheat heads were inoculated with the conidia of F. graminearum containing $100 \mu \mathrm{g} / \mathrm{mL}$ of mycosubtilin. After 7 days, the diseased wheat kernels turned gray, while the healthy kernels remained green. The wheat heads treated with mycosubtilin remained healthy, and the average proportion of symptomatic spikelets (PSS) was reduced to $12.87 \%$ compared with the control. This indicated that mycosubtilin can reduce the pathogenicity of F. graminearum to wheat heads (Figure 5A,B). We also injected the conidia of F. verticillioides into wounded maize kernels and observed a reduction of aerial hyphae in the $100 \mu \mathrm{g} / \mathrm{mL}$ mycosubtilin treatment compared to the untreated control (Figure 5C). These results showed a $100 \mu \mathrm{g} / \mathrm{mL}$ mycosubtilin treatment could effectively inhibit the infection of F. graminearum and F. verticillioides on wheat and maize, respectively. Additionally, wheat heads and maize kernels treated with mycosubtilin alone remained healthy, suggesting that the use of mycosubtilin would not produce obvious detrimental side effects in plants. 

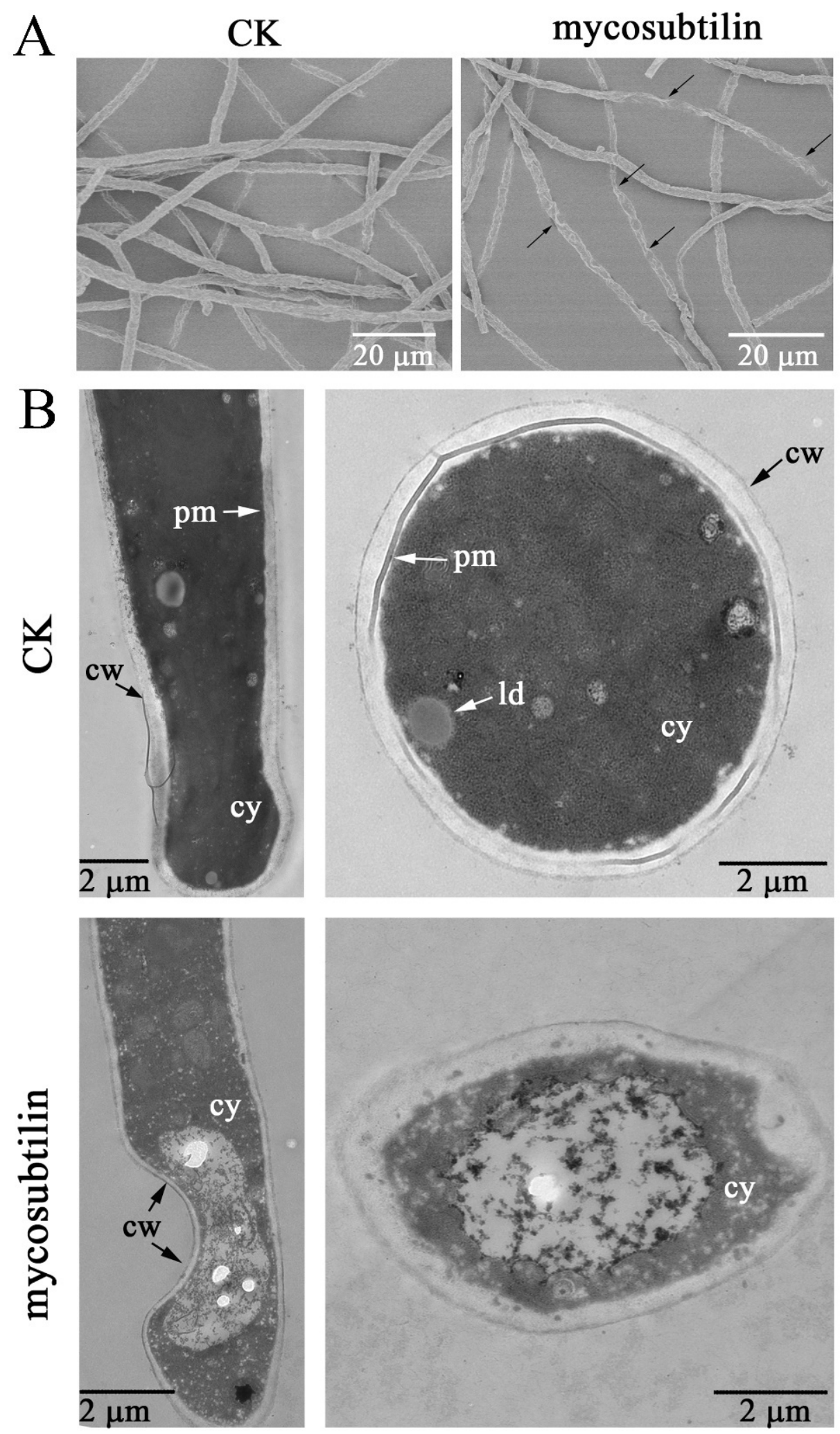

Figure 3. Morphology changes in F. graminearum hyphae induced by $20 \mu \mathrm{g} / \mathrm{mL}$ of mycosubtilin, detected via SEM (A) and TEM (B). CK, 25\% (vol/vol) methanol. cw, cell wall; cy, cytoplasm; pm, plasma membrane; ld, lipid droplets. 
A

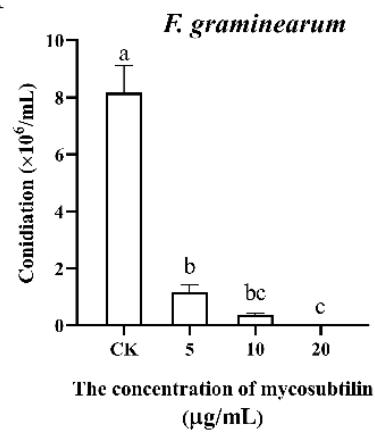

$\mathrm{C}$

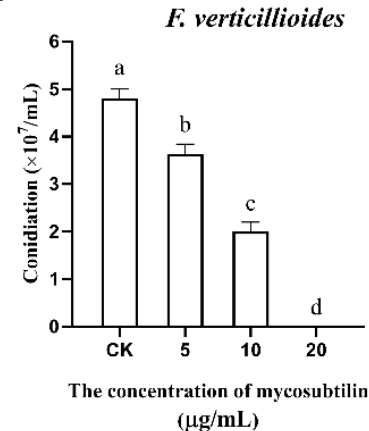

B

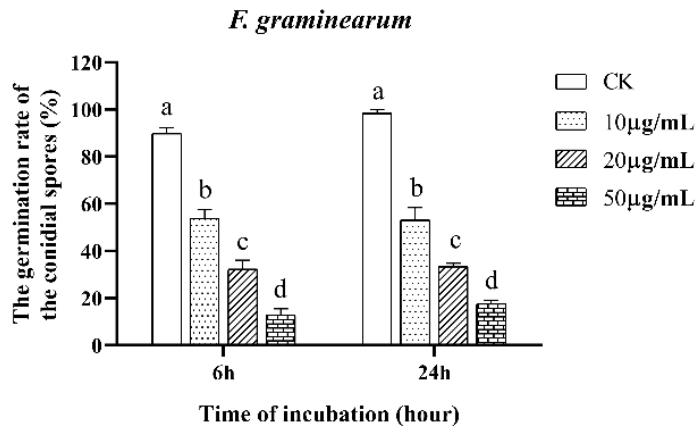

$\mathrm{D}$

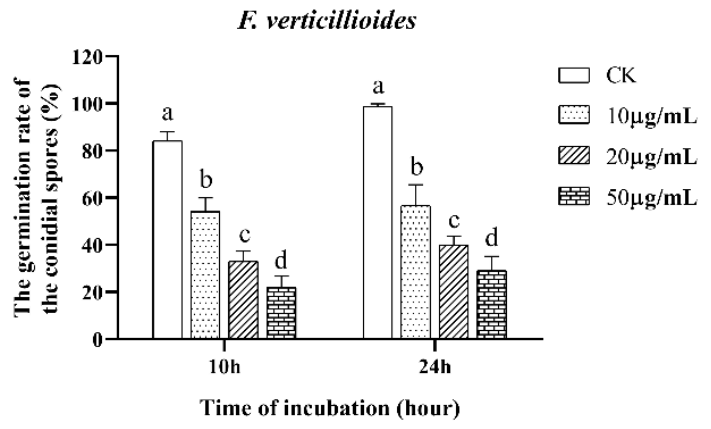

Figure 4. Effects of mycosubtilin on conidial formation and germination of $F$. graminearum $(\mathbf{A}, \mathbf{B})$ and $F$. verticillioides (C,D). CK, $25 \%(\mathrm{vol} / \mathrm{vol})$ methanol. Data were analyzed by one-way ANOVA, followed by Duncan's multiple range test. Line bars represent standard errors of three repeated experiments. Letters $\mathrm{a}, \mathrm{b}, \mathrm{c}$, and $\mathrm{d}$ above the columns represent statistically significant differences $(\alpha=0.05)$.

\subsection{Mycosubtilin Inhibited Mycotoxin Production of F. graminearum and F. verticillioides}

DON produced by F. graminearum and the FBs produced by F. verticillioides are the most hazardous and the most studied mycotoxins. Consequently, a mycotoxin production assay was conducted to evaluate the effect of mycosubtilin on plural mycotoxins. Sterilized wheat/maize kernels were incubated with conidial suspensions of $F$. graminearum /F. verticillioides containing mycosubtilin. After 30 days of incubation, plural mycotoxins were extracted and quantified by LC-MS analysis. At a concentration of $50 \mu \mathrm{g} / \mathrm{mL}$ of mycosubtilin, the mycotoxin was detected by LC-MS, and after exposure to $100 \mu \mathrm{g} / \mathrm{mL}$ of mycosubtilin, fungal growth was completely inhibited, and the samples could not produce mycotoxin.

These results demonstrated that treatment with $50 \mu \mathrm{g} / \mathrm{mL}$ of mycosubtilin could reduce DON and FBs production by $F$. graminearum and F. verticillioides, respectively (Figure 6). 


\subsection{Mycosubtilin Downregulated the Expression Level of DON and FBs-Related Genes}

In F. graminearum, 15 Tri genes are responsible for the biosynthesis of trichothecene, while in F. verticillioides, the Fum gene cluster is involved in fumonisin production [11,30]. To study the mechanism underlying the reduction of DON and FBs production caused by mycosubtilin, the expression of Tri genes (FgTri5, FgTri10, and FgTri12) and Fum genes (FvFum1, FvFum7, and FvFum8) was analyzed. The expression level of six regulating genes was significantly downregulated by $50 \mu \mathrm{g} / \mathrm{mL}$ mycosubtilin treatment, suggesting that mycosubtilin can negatively influence the DON and FBs biosynthesis pathways (Figure 7).

A

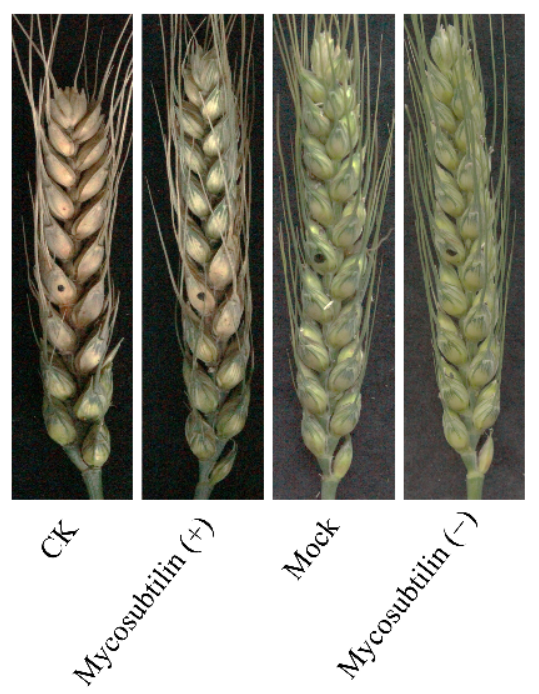

B

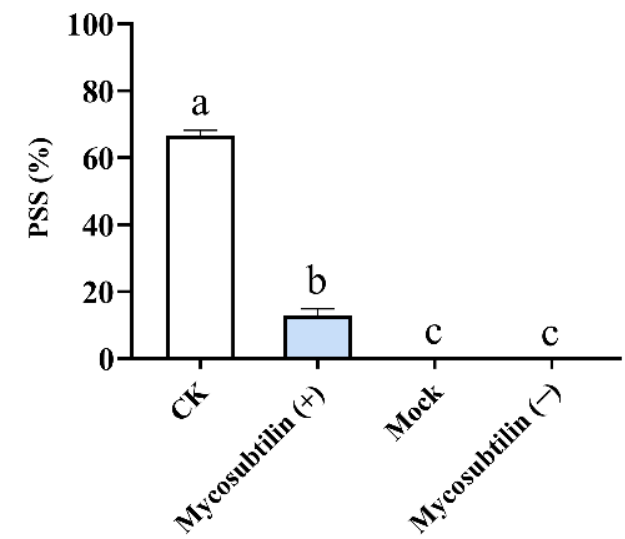

C

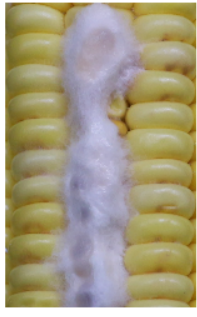

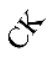
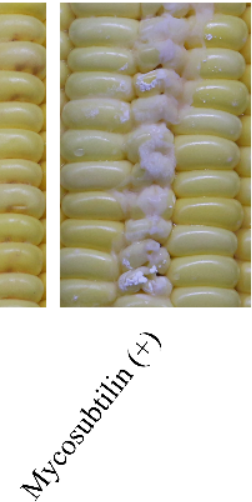
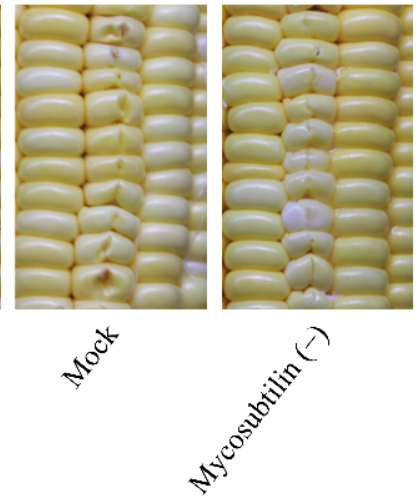

Figure 5. Effects of mycosubtilin on the pathogenicity of $F$. graminearum $(\mathbf{A}, \mathbf{B})$ and $F$. verticillioides (C) in plants. Mock, solution with $25 \%(\mathrm{vol} / \mathrm{vol})$ methanol; CK, a conidial suspension with $25 \%$ methanol; Mycosubtilin (+), a conidial suspension with $100 \mu \mathrm{g} / \mathrm{mL}$ of mycosubtilin; Mycosubtilin (-), $\mathrm{ddH}_{2} \mathrm{O}$ containing $100 \mu \mathrm{g} / \mathrm{mL}$ of mycosubtilin. (A) F. graminearum $\mathrm{PH}-1$ was used to infect wheat heads. Black dots on the wheat ears represent the inoculation site; (B) the pathogenicity of F. graminearum was measured by the average PSS. Data were analyzed by a one-way ANOVA, followed by Duncan's multiple range test. Line bars represent standard errors of three repeated experiments. Letters $\mathrm{a}, \mathrm{b}$, and $\mathrm{c}$ above the columns represent statistically significant differences $(\alpha=0.05)$; (C) F. verticillioides 7600 was used to infect maize kernels. 
A

$\mathrm{C}$
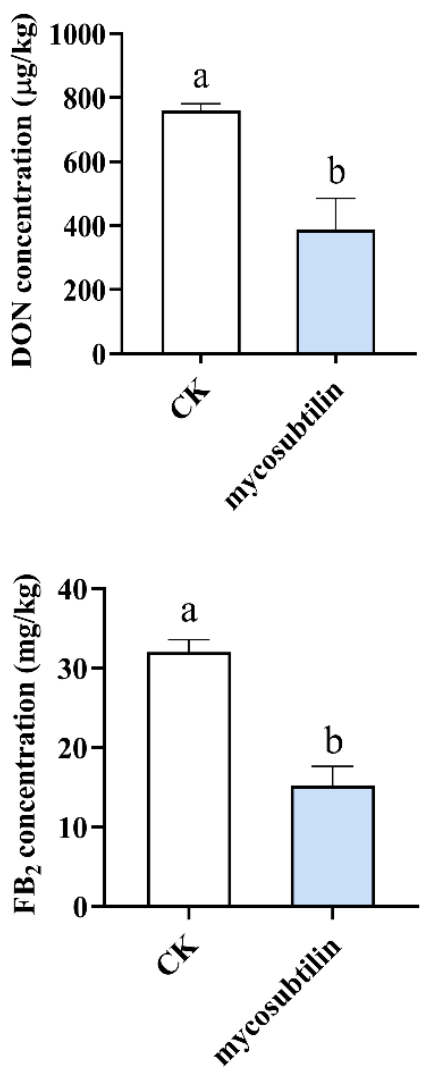

B

$\mathrm{D}$
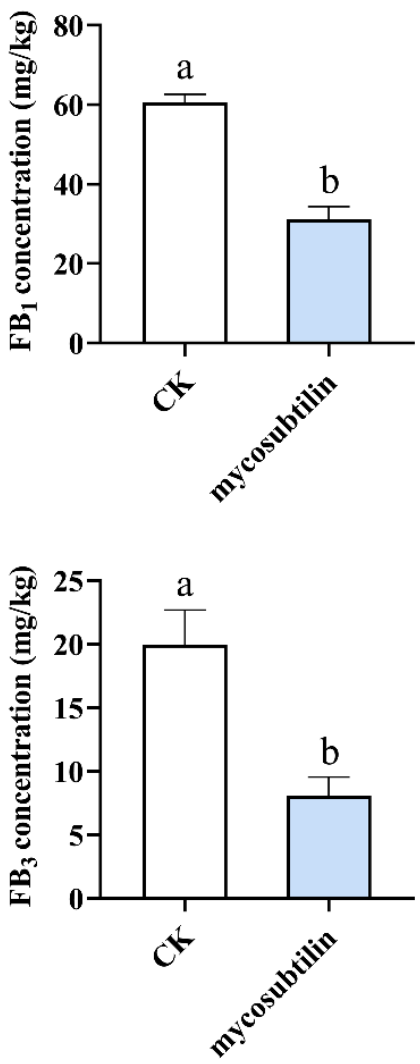

Figure 6. Effects of $50 \mu \mathrm{g} / \mathrm{mL}$ mycosubtilin treatment on mycotoxin biosynthesis. Content of DON (A) in wheat kernels and that of $\mathrm{FB}_{1}(\mathbf{B}), \mathrm{FB}_{2}(\mathbf{C})$, and $\mathrm{FB}_{3}(\mathbf{D})$ in maize kernels after 30 days of incubation, with or without mycosubtilin. CK, 25\% ( vol/vol) methanol. Data were analyzed by one-way ANOVA. Line bars represent standard errors of three repeated experiments. Letters $a$ and $b$ above the columns represent statistically significant differences $(\alpha=0.05)$.

A

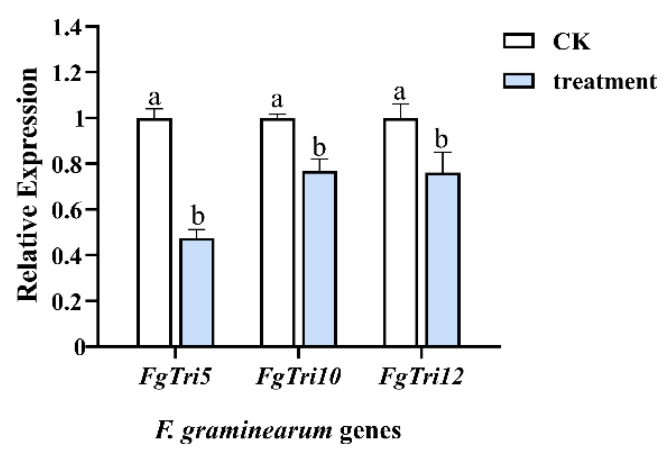

B

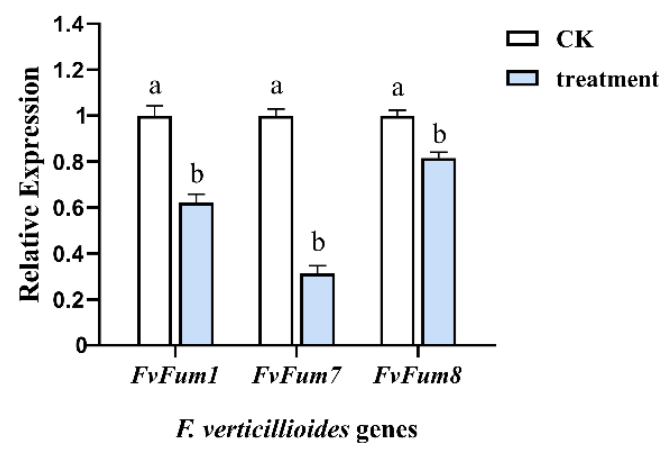

Figure 7. The expression level of DON-related genes of F. graminearum (A) and FBs-related genes of F. verticillioides (B). CK, $25 \%(\mathrm{vol} / \mathrm{vol})$ methanol. Values were normalized to the levels of the actin gene as an internal reference. Data were analyzed by one-way ANOVA. Line bars represent standard errors of three repeated experiments. Letters a and $b$ above the columns represent statistically significant differences $(\alpha=0.05)$.

\section{Discussion}

Lipopeptides (LPs) are important in Bacillus spp. biocontrol of phytopathogens [19]. They offer possible control of plant pathogen infections because they are selectively nontoxic, highly stable, and ecofriendly [31]. The fengycin and iturin families are strong inhibitors of fungal pathogens. Previous studies demonstrated that fengycin has an- 
tagonistic action against F. graminearum and S. sclerotiorum in vitro and in vivo [20,23]; bacillomycin D synthesized by Bacillus velezensis FZB42 had significant inhibitory activity against F. graminearum [29]. Mycosubtilin, a member of the iturin family, is characterized by a $\beta$-amino fatty acid linked to heptapeptide with a LDDLLDL chiral sequence in Asn-Tyr-Asn-Gln-Pro-Ser-Asn order [27]. Mycosubtilin exhibits antagonistic activities against Bremia lactucae [32], Fusarium oxysporum [33], and S. cerevisiae [34], suggesting its potential as an antifungal agent. However, the effects of mycosubtilin on F. graminearum or F. verticillioides have not yet been studied. In the present study, mycosubtilin was isolated from B. subtilis ATCC6633, and it showed a strong and durative inhibitory effect against F. graminearum and F. verticillioides in an antifungal assay (Figures 1 and 2, Figures S1 and S2).

To explore the actions of mycosubtilin, SEM and TEM were used to observe the ultrastructural changes in the hyphae of $F$. graminearum PH-1. The SEM result indicated that $20 \mu \mathrm{g} / \mathrm{mL}$ of mycosubtilin had an adverse effect on the hyphal structure, while TEM analysis revealed that mycosubtilin can disrupt the cell wall and plasma membrane (Figure 3). These findings were consistent with previous research reporting that bacillomycin $\mathrm{D}$, as another member of the iturin family, causes morphological changes in the hyphae and conidia of F. graminearum [29]. In other examples, LPs produced by B. subtilis B1 led to the plasmolysis and shrinkage of Lasiodiplodia theobromae [35]; iturins caused defects in the cell wall integrity of Verticillium dahlia [36], consistent with our results. The action of mycosubtilin damages hyphal cells by interacting with the ergosterol present in the membranes of fungi [37]. This suggests a possible reason for the morphological changes induced by mycosubtilin on the hyphal structure of F. graminearum.

F. graminearum and F. verticillioides infect crop plants by relying on sporulation and germination during their asexual life cycle. In the present study, fungal spore formation and germination were suppressed in vitro by mycosubtilin (Figure 4). To investigate the in-vivo inhibitory effect of mycosubtilin against these two pathogens, an in planta experiment was conducted, and it demonstrated that $100 \mu \mathrm{g} / \mathrm{mL}$ of mycosubtilin reduced the virulence of $F$. graminearum in wheat heads. Compared to the control, the average proportion of symptomatic spikelets (PSS) decreased sharply upon treatment with mycosubtilin (Figure 5A,B). These results were similar to the previous study wherein F. graminearuminfected wheat spikes were treated with $90 \mu \mathrm{g} / \mathrm{mL}$ of fengycin [20]. When the conidial spores of $F$. verticillioides were used to infect maize kernels, a distinct reduction of symptoms was observed relative to the control (Figure 5C), indicating the inhibitory effect of mycosubtilin on conidial spores. The adverse effects on hyphae caused by another iturin, bacillomycin $\mathrm{D}(90 \mu \mathrm{g} / \mathrm{mL})$, reduced the infection of corn silks by F. graminearum hyphae, suggesting a potential function of mycosubtilin. Taken together, the pathogenicity tests of F. graminearum and F. verticillioides showed the substantial antagonistic activity of mycosubtilin against these fungi in vivo. The results suggest that mycosubtilin has potential for development as an antifungal agent. The dose of mycosubtilin used in the current study was similar to the concentrations of other LPs of Bacillus tested against F. graminearum $[20,29]$.

However, the method of inoculating fungus together with mycosubtilin does not simulate a natural field situation. Additional experiments should be conducted to study the preventative effect of mycosubtilin against $F$. graminearum and $F$. verticillioides. Prior to field application, the protective effect of mycosubtilin on other wheat/maize cultivars against different strains of $F$. graminearum / F. verticillioides should also be studied.

DON is one of the most common mycotoxins associated with F. graminearum, and DON biosynthesis was inhibited by mycosubtilin (Figure 6A). Our results are supported by a previous study, which reported that iturin A, fengycin, and surfactin from B. amyloliquefaciens JCK-12 act together to decrease the yield of trichothecenes by F. graminearum [38]. However, Gu et al. reported that bacillomycin D can stimulate DON biosynthesis by activating the phosphorylation of $F g H O G 1$ and $F g M G V 1$, which is in contrast to our findings [29]. We hypothesized that the distinct function of mycosubtilin and bacillomycin D in DON production is due to the different amino acid residues of heptapeptide. Fumonisins are the most 
abundant mycotoxins found in maize and are often associated with toxicoses in humans and livestock. FBs with a 20-carbon backbone have high toxicity and carcinogenicity. We also noted that mycosubtilin caused a large reduction in FBs production in maize kernels infected by F. verticillioides (Figure 6B-D), which was similar to results showing that fengycin can downregulate the expression levels of Fum 1 and Fum 8 and inhibit the production of FB1 by F. verticillioides [39]. The qRT-PCR analysis showed that the expression level of FgTri/FvFum genes was downregulated after mycosubtilin treatment, demonstrating the inhibitory effect of mycosubtilin on DON and FBs biosynthesis at the transcriptional level (Figure 7). In summary, we report that mycosubtilin produced by B. subtilis ATCC6633 is a candidate compound for the control of the diseases and mycotoxin accumulation caused by F. graminearum and F. verticillioides.

\section{Materials and Methods}

\subsection{Culture Conditions of Bacteria and Fungi}

B. subtilis ATCC6633, F. graminearum PH-1, and F. verticillioides 7600 strains were stored in our laboratory. B. subtilis ATCC6633 strain was cultivated in Luria-Bertani (LB) medium at $37^{\circ} \mathrm{C}$, while the fungal species were cultivated in potato dextrose agar (PDA) at $25^{\circ} \mathrm{C}$. For the preparation of conidia suspension, fresh mycelia $(50 \mathrm{mg})$ of $F$. graminearum PH-1 and F. verticillioides 7600 were inoculated in $20 \mathrm{~mL}$ liquid carboxymethyl cellulose (CMC) medium $\left(1 \mathrm{~g} \mathrm{NH}_{4} \mathrm{NO}_{3}, 1 \mathrm{~g} \mathrm{KH}_{2} \mathrm{PO}_{3}, 0.5 \mathrm{~g} \mathrm{MgSO}_{4} \cdot 7 \mathrm{H}_{2} \mathrm{O}, 1 \mathrm{~g}\right.$ yeast extract, $15 \mathrm{~g}$

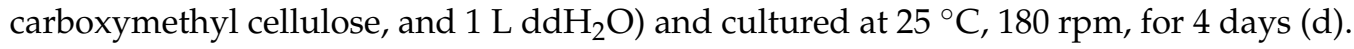

\subsection{Antifungal Activity Assay}

The antagonistic activity of purified mycosubtilin was studied on a PDA plate. A 6-mm-diameter agar disk containing mycelia was placed at the center of a PDA plate. Subsequently, 7-mm-diameter wells were made with a sterilized bore at $3 \mathrm{~cm}$ from the center. Then, $10 \mu \mathrm{L}$ of purified mycosubtilin was added to the wells and incubated at $25^{\circ} \mathrm{C}$ for $6 \mathrm{~d}$. The plates were then photographed, and the mycelial length of fungus was measured. The percentage of inhibition was calculated by using the following formula:

$$
\text { Inhibition rate }(\%)=[(\mathrm{C}-\mathrm{T}) \times 100) / \mathrm{C}]
$$

where control $(\mathrm{C})$ and treatment $(\mathrm{T})$ are the mycelial length $(\mathrm{cm})$ of the fungus from central mycelial plug to side edge of CK and mycosubtilin, respectively. The experiment was repeated three times, and three replicates were performed for each experiment.

\subsection{Purification and Identification of Mycosubtilin from B. subtilis ATCC6633}

A single colony of ATCC 6633 was inoculated in $20 \mathrm{~mL}$ of LB medium and cultured at $37^{\circ} \mathrm{C}$ for $24 \mathrm{~h}$. Then, $2 \mathrm{~mL}$ of this culture was transferred to $200 \mathrm{~mL}$ of LB medium, followed by incubation for $48 \mathrm{~h}$ at $37^{\circ} \mathrm{C}$. After the first $24 \mathrm{~h}$ of incubation, $2 \mathrm{~g}$ of sterilized Amberlite Resin XAD16N (Lot BCCD6602, Sigma, St. Louis, MO, USA) was added to the culture to absorb compounds of molecular mass $<3 \mathrm{kDa}$. The sample was then centrifuged at $10,000 \times \mathrm{rpm}$ for $20 \mathrm{~min}$, and the supernatant was discarded. The precipitates containing XAD16N were re-dissolved with $10 \mathrm{~mL}$ methanol and incubated at $37^{\circ} \mathrm{C}$ for $4 \mathrm{~h}$. Subsequently, the sample was filtered through double filter paper and concentrated using a rotary evaporator. The crude extract was obtained by suspending samples in methanol and filtration $(0.22 \mu \mathrm{m}$, Nylon).

The crude extract was added to a semipreparative high-performance liquid chromatograph (HPLC, Waters, Milford, MA, USA) equipped with a reverse-phase column (ZORBAX SB-C18, $9.4 \times 250 \mathrm{~mm}, 5 \mu \mathrm{m}$, Agilent, Palo Alto, CA, USA). The mobile phase comprised A (HPLC-grade acetonitrile with $0.1 \%$ trifluoroacetic acid) and B (Milli-Q water with $0.1 \%$ trifluoroacetic acid). The gradient elution procedure used $30-95 \%$ acetonitrile (ACN) for $30 \mathrm{~min}$ and then was held at $95 \%$ for $10 \mathrm{~min}$. Mycosubtilin was eluted between $12-15 \mathrm{~min}$. The detection was made under UV absorption at $214 \mathrm{~nm}$, and the flow rate was $4 \mathrm{~mL} / \mathrm{min}$. 
Purified mycosubtilin was verified via ultra-performance liquid chromatographymass spectrometry (UPLC-MS, G2 QTof-XS, Waters, Milford, MA, USA) with a UPLC column $\left(\mathrm{C}_{18}, 2.1 \times 50 \mathrm{~mm}, 1.7 \mu \mathrm{m}\right.$, Waters, Milford, MA, USA). Mobile phase A was ACN with $0.1 \%(v o l / v o l)$ formic acid, while mobile phase B was Milli-Q water (Millipore, Billerica, MA, USA). The samples were eluted from $2-98 \%$ ACN in 8 min and held at $98 \%$ for $2 \mathrm{~min}$ with the flow rate at $0.4 \mathrm{~mL} / \mathrm{min}$. The injection volume was $1 \mu \mathrm{L}$, and a photo-diode-arraydetector was used to monitor the range of $200-400 \mathrm{~nm}$. Mass spectrometry was carried out via an electrospray ionization (ESI) source operating in the positive ion mode. The MS parameters were as follows: source voltage $2.5 \mathrm{kV}$, collision energy $40 \mathrm{eV}$, and acquisition range $100-1500 \mathrm{~m} / \mathrm{z}$. The purified mycosubtilin was identified by comparing detected molecular ion peaks with those previously reported [28]. The purity of mycosubtilin was calculated, based on the peak area, as $94.3 \%$.

\subsection{Hyphal Morphological Observation via Scanning Electron Microscope (SEM) and Transmission Electron Microscope (TEM)}

To observe the morphology changes of hyphae from F. graminearum $\mathrm{PH}-1$ treated with mycosubtilin $(20 \mu \mathrm{g} / \mathrm{mL})$ at the ultrastructural level, TEM and SEM were used. Hyphae of F. graminearum were collected after $12 \mathrm{~h}$ treatment with mycosubtilin. For SEM, the samples were prefixed with $2.5 \%$ glutaraldehyde and followed by rinsing in triplicate using $100 \mathrm{mM}$ phosphate buffer. Subsequently, the samples were postfixed for $3 \mathrm{~h}$ in $1 \%$ osmium tetroxide and dehydrated using an ethanol gradient. After that, the samples were coated with gold particles and observed via Hitachi S-3000N scanning electron microscope at voltage $5 \mathrm{kV}$ (Hitachi, Tokyo, Japan). As for TEM analysis, the prefixed cells were embedded in Epon 812, sectioned using an ultra-microtome, and examined with a Hitachi H-600 transmission electron microscope.

\subsection{The Assay of Conidia Formation and Germination}

For the spore formation assay, the mycelia of F. graminearum (or F. verticillioides) was inoculated in CMC medium containing different concentrations of mycosubtilin $(5,10$, and $20 \mu \mathrm{g} / \mathrm{mL})$, and $25 \%(\mathrm{vol} / \mathrm{vol})$ methanol was used as the control. After $3 \mathrm{~d}$ of incubation at $25^{\circ} \mathrm{C}$ and $180 \mathrm{rpm}$, the number of conidia was measured using a hemacytometer. The experiment was repeated three times with three replicates.

To determine the germination rate of fungal spores, $1 \mathrm{~mL}$ of conidial suspension $\left(10^{6}\right.$ conidia $\left./ \mathrm{mL}\right)$ with different concentrations of mycosubtilin $(10,20$, and $50 \mu \mathrm{g} / \mathrm{mL})$ were cultured at $25^{\circ} \mathrm{C}$ and $180 \mathrm{rpm}$. The incubation time for F. graminearum is 6 and $24 \mathrm{~h}$, while that for F. verticillioides is 10 and $24 \mathrm{~h}$. A spore was considered germinated if the germ tube reached at least half the length of the spore. The germination rate was quantified by counting at least 200 conidia for each replication by microscopy. As the control, $25 \%(\mathrm{vol} / \mathrm{vol})$ methanol was used. The experiment was repeated three times with three replicates.

Germination and conidial morphology of each sample were examined using the DIC mode of an OLYMPUS-IX71 inverted microscope (Tokyo, Japan). The conidia that had been treated with $10 \mu \mathrm{g} / \mathrm{mL}$ mycosubtilin for $3 \mathrm{~d}$ were assayed using propidium iodide (Solarbio, Beijing, China). Fungal cells were imaged using an OLYMPUS-IX71 inverted microscope, and those with damaged membranes showed red fluorescence.

\subsection{Pathogenicity Assay}

To detect the effect of mycosubtilin on the pathogenicity of $F$. graminearum and F. verticillioides in wheat heads and maize kernels, corresponding greenhouse experiments were conducted. The experiment consisted of four treatments: (1) Mock, inoculated with $10 \mu \mathrm{L}$ of $25 \%$ (vol/vol) methanol only; (2) CK, inoculated with $10 \mu \mathrm{L}$ conidial suspension with 25\% methanol; (3) Mycosubtilin (+), treated with $10 \mu \mathrm{L}$ conidial suspension containing

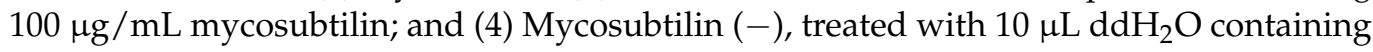
$100 \mu \mathrm{g} / \mathrm{mL}$ mycosubtilin. The concentration of conidial suspension used in greenhouse experiments was $10^{6}$ conidia $/ \mathrm{mL}$. 
For wheat heads, wheat that reached the anthesis stage was used. The fifth spikelet from the base of the spike was drop inoculated with different solutions of treatment. The plants were kept in the greenhouse with condition as follows: $25^{\circ} \mathrm{C}, 100 \%$ humidity, and $12 \mathrm{~h}$ of the daylight period. After $7 \mathrm{~d}$, different samples were photographed, and the average proportion of symptomatic spikelets (PSS) was measured [40]. The experiment was repeated three times, and fourteen replicates were performed for each treatment.

For maize kernels, their surface was first sterilized by a spray of $75 \%$ ethanol and $\mathrm{ddH}_{2} \mathrm{O}$. After that, the maize kernels were scratched using sterilized toothpicks and inoculated under different treatments. Three replicates were used for each treatment. Maize kernels were incubated under $25^{\circ} \mathrm{C}, 100 \%$ humidity, for $7 \mathrm{~d}$, in a 12-h daylight period [41]. The experiment was repeated three times.

\subsection{Determination of Mycotoxin Production}

A total of $50 \mathrm{~g}$ of detached wheat (or maize) kernels were sterilized and inoculated with $1 \mathrm{~mL}$ conidial suspension $\left(10^{6}\right.$ conidial $/ \mathrm{mL}$ ) of F. graminearum (or F. verticillioides) containing $50 \mu \mathrm{g} / \mathrm{mL}$ purified mycosubtilin. As the control, $1 \mathrm{~mL}$ conidial suspension with $25 \%$ $(\mathrm{vol} / \mathrm{vol})$ methanol was used. After incubation at $25^{\circ} \mathrm{C}$ for $30 \mathrm{~d}$, mycotoxin was extracted as described previously [42-44] and analyzed using a HPLC-mass spectrometer/mass spectrometer (HPLC-MS/MS) system (Shimadzu 30A LC system coupled to a Triple Quad 6500 plus, Sciex, Framingham, MA, USA) to determine the amount of mycotoxin in the corresponding sample. The experiment was repeated thrice, and three replicates were performed for each treatment.

\subsection{RNA Extraction and Expression Analysis by qRT-PCR}

For total RNA extraction, mycelia of F. graminearum PH-1 (or F. verticillioides 7600) were inoculated in a 50-mL flask containing $20 \mathrm{~mL}$ of potato dextrose broth (PDB) at $25{ }^{\circ} \mathrm{C}$ for $36 \mathrm{~h}$. Either $50 \mu \mathrm{g} / \mathrm{mL}$ mycosubtilin or $25 \%$ methanol (set as a control treatment) was added into the flask for another $2 \mathrm{~h}$. Followed by the manufacturer's guidelines, the total RNA of each sample was extracted from the harvested mycelia using the TaKaRa RNAiso reagent (TaKaRa Biotechnology Co., Ltd., Dalian, China). Reverse transcription was conducted by using HiScript III RT SuperMix (Vazyme Biotech Co., Ltd., Nanjing, China). The expression level of Tri and Fum genes was investigated in a 7500 fast real-time PCR detector by using HiScript II One Step qRT-PCR SYBR Green Kit (Vazyme Biotech Co., Ltd. Nanjing, China). The conditions of qRT-PCR consisted of hold at $95{ }^{\circ} \mathrm{C}$ for $30 \mathrm{~s}$, followed by 40 cycles of $5 \mathrm{~s}$ at $95^{\circ} \mathrm{C}$ and $30 \mathrm{~s}$ at $60^{\circ} \mathrm{C}$. The FgActin and FvActin gene were amplified as housekeeping genes for normalization. The expression analysis was carried out with the $\triangle \Delta C T$ method using the 7500 system SDS software. Primers used for qRT-PCR are listed in Table S1. The experiment was repeated thrice independently, with three replicates for each treatment.

Supplementary Materials: The following are available online at https:/ /www.mdpi.com/article/10.3 390/toxins13110791/s1, Table S1: Primers used in this study; Figure S1: In the in vitro antifungal assay, percentage inhibition of mycosubtilin against $F$. graminearum and F. verticillioides after incubation of 3 days and 6 days was calculated. Data were analyzed by one-way ANOVA. Line bars represent standard errors of three repeated experiments. Same letter of a above the columns represents no statistically significant differences $(\alpha=0.05)$; Figure S2: In the in vitro antifungal assay, percentage inhibition of mycosubtilin $(20,50,100,250$, and $500 \mu \mathrm{g} / \mathrm{mL})$ against $F$. graminearum after incubation of 3 days and 6 days was calculated. Data were analyzed by one-way ANOVA, followed by Duncan's multiple range test. Line bars represent standard errors of three repeated experiments. Letters a, $b$ and $c$ above the columns represent statistically significant differences $(\alpha=0.05)$, while similar letters $\mathrm{ab}$ and $\mathrm{bc}$ represent no statistically significant differences; Figure S3: The expression level of conidiation-related genes (fgsg_06774,fgsg_02471,fgsg_10166,fveg_05214,fveg_05267,fveg_05331) of F. graminearum (A) and F. verticillioides (B). CK (control), 25\% (vol/vol) methanol. Treatment, $50 \mu \mathrm{g} / \mathrm{mL}$ mycosubtilin. Values were normalized to the levels of the actin gene as an internal reference. Data were analyzed by one-way ANOVA. Line bars represent standard errors of three repeated experiments. Letters $\mathrm{a}$ and $\mathrm{b}$ above the columns represent statistically significant differences 
$(\alpha=0.05)$; Figure S4: Morphology changes in conidia of F. graminearum and F. verticillioides induced by $10 \mu \mathrm{g} / \mathrm{mL}$ of mycosubtilin, detected by inverted microscope. CK, $25 \%$ ( $\mathrm{vol} / \mathrm{vol})$ methanol; Figure S5: Inhibitory activity of different concentrations of mycosubtilin $(10,20$ and $50 \mu \mathrm{g} / \mathrm{mL})$ on conidial germination of F. graminearum and F. verticillioides, detected by inverted microscope. CK, 25\% (vol/vol) methanol; Figure S6: Viablity of F. graminearum (A) and F. verticillioides (B) conidia after treatment with $10 \mu \mathrm{g} / \mathrm{mL}$ of mycosubtilin for 3 days. Fungal cells with damaged membranes showed red fluorescence. CK, $25 \%(\mathrm{vol} / \mathrm{vol})$ methanol.

Author Contributions: Conceptualization, H.W. and Q.G.; Experiment and data analysis, C.Y., X.Z., M.Z., Y.G., J.X. and J.S.; writing-original draft preparation, C.Y., X.L. and Q.A.; writing-review and editing, X.G., M.S.R.M. and Q.G. All authors have read and agreed to the published version of the manuscript.

Funding: This research was funded by the National Key R\&D Program of China (2018YFE0206000), the Natural Science Foundation for Excellent Youth Scholars of Jiangsu Province, China (BK20160719), the National Natural Science Foundation of China (32072364), the Natural Science Foundation of Jiangsu Province, China (BK20181322), and Fundamental Research Funds for the Central Universities (JCQY201904).

Institutional Review Board Statement: Not applicable.

Informed Consent Statement: Not applicable.

Data Availability Statement: Data are available upon request; please contact the contributing authors.

Conflicts of Interest: The authors declare no conflict of interest.

\section{References}

1. Ma, L.J.; Geiser, D.M.; Proctor, R.H.; Rooney, A.P.; O’Donnell, K.; Trail, F.; Gardiner, D.M.; Manners, J.M.; Kazan, K. Fusarium Pathogenomics. In Annual Review of Microbiology; Gottesman, S., Ed.; Annual Reviews: Palo Alto, CA, USA, 2013; Volume 67, pp. 399-416.

2. Chen, Y.; Wang, W.X.; Zhang, A.F.; Gu, C.Y.; Zhou, M.G.; Gao, T.C. Activity of the fungicide JS399-19 against Fusarium head blight of wheat and the risk of resistance. Agric. Sci. China 2011, 10, 1906-1913. [CrossRef]

3. Goswami, R.S.; Kistler, H.C. Heading for disaster: Fusarium graminearum on cereal crops. Mol. Plant Pathol. 2004, 5, 515-525. [CrossRef]

4. Munkvold, G.P. Cultural and genetic approaches to managing mycotoxins in maize. Annu. Rev. Phytopathol. 2003, 41, 99-116. [CrossRef] [PubMed]

5. Nelson, P.E. Taxonomy and biology of Fusarium moniliforme. Mycopathologia 1992, 117, 29-36. [CrossRef] [PubMed]

6. Da Rocha, M.E.B.; Freire, F.D.O.; Maia, F.B.F.; Guedes, M.I.F.; Rondina, D. Mycotoxins and their effects on human and animal health. Food Control 2014, 36, 159-165. [CrossRef]

7. Pestka, J.J.; Smolinski, A.T. Deoxynivalenol: Toxicology and potential effects on humans. J. Toxicol. Environ. Health-Part B-Crit. Rev. 2005, 8, 39-69. [CrossRef] [PubMed]

8. Munkvold, G.P. Fusarium species and their associated mycotoxins. In Mycotoxigenic Fungi: Methods and Protocols; Moretti, A., Susca, A., Eds.; Springer: Berlin/Heidelberg, Germany, 2017; Volume 1542, pp. 51-106.

9. Ferrigo, D.; Raiola, A.; Causin, R. Fusarium toxins in cereals: Occurrence, legislation, factors promoting the appearance and their management. Molecules 2016, 21, 627. [CrossRef] [PubMed]

10. McCormick, S.P.; Stanley, A.M.; Stover, N.A.; Alexander, N.J. Trichothecenes: From simple to complex mycotoxins. Toxins 2011, 3, 802-814. [CrossRef]

11. Chen, Y.; Kistler, H.C.; Ma, Z.H. Fusarium graminearum trichothecene mycotoxins: Biosynthesis, regulation, and management. In Annual Review of Phytopathology; Leach, J.E., Lindow, S.E., Eds.; Annual Reviews: Palo Alto, CA, USA, 2019; Volume 57, pp. 15-39.

12. Pestka, J.J. Deoxynivalenol: Mechanisms of action, human exposure, and toxicological relevance. Arch. Toxicol. 2010, 84, 663-679. [CrossRef]

13. Theumer, M.G.; Canepa, M.C.; Lopez, A.G.; Mary, V.S.; Dambolena, J.S.; Rubinstein, H.R. Subchronic mycotoxicoses in Wistar rats: Assessment of the in vivo and in vitro genotoxicity induced by fumonisins and aflatoxin B-1, and oxidative stress biomarkers status. Toxicology 2010, 268, 104-110. [CrossRef] [PubMed]

14. Wang, J.; Liu, J.; Chen, H.; Yao, J. Characterization of Fusarium graminearum inhibitory lipopeptide from Bacillus subtilis IB. Appl. Microbiol. Biotechnol. 2007, 76, 889-894. [CrossRef]

15. Chen, X.H.; Koumoutsi, A.; Scholz, R.; Eisenreich, A.; Schneider, K.; Heinemeyer, I.; Morgenstern, B.; Voss, B.; Hess, W.R.; Reva, O.; et al. Comparative analysis of the complete genome sequence of the plant growth-promoting bacterium Bacillus amyloliquefaciens FZB42. Nat. Biotechnol. 2007, 25, 1007-1014. [CrossRef] [PubMed]

16. Glick, B.R. The enhancement of plant-growth by free-living bacteria. Can. J. Microbiol. 1995, 41, 109-117. [CrossRef] 
17. Bhattacharyya, P.N.; Jha, D.K. Plant growth-promoting rhizobacteria (PGPR): Emergence in agriculture. World J. Microbiol. Biotechnol. 2012, 28, 1327-1350. [CrossRef] [PubMed]

18. Stein, T. Bacillus subtilis antibiotics: Structures, syntheses and specific functions. Mol. Microbiol. 2005, 56, 845-857. [CrossRef] [PubMed]

19. Raaijmakers, J.M.; de Bruijn, I.; Nybroe, O.; Ongena, M. Natural functions of lipopeptides from Bacillus and Pseudomonas: More than surfactants and antibiotics. FEMS Microbiol. Rev. 2010, 34, 1037-1062. [CrossRef] [PubMed]

20. Hanif, A.; Zhang, F.; Li, P.P.; Li, C.C.; Xu, Y.J.; Zubair, M.; Zhang, M.X.; Jia, D.D.; Zhao, X.Z.; Liang, J.G.; et al. Fengycin produced by Bacillus amyloliquefaciens FZB42 inhibits Fusarium graminearum growth and mycotoxins biosynthesis. Toxins 2019, 11, 295. [CrossRef] [PubMed]

21. Ongena, M.; Jacques, P. Bacillus lipopeptides: Versatile weapons for plant disease biocontrol. Trends Microbiol. 2008, 16, 115-125. [CrossRef]

22. Toure, Y.; Ongena, M.; Jacques, P.; Guiro, A.; Thonart, P. Role of lipopeptides produced by Bacillus subtilis GA1 in the reduction of grey mould disease caused by Botrytis cinerea on apple. J. Appl. Microbiol. 2004, 96, 1151-1160. [CrossRef]

23. Farzand, A.; Moosa, A.; Zubair, M.; Khan, A.R.; Massawe, V.C.; Tahir, H.A.S.; Sheikh, T.M.M.; Ayaz, M.; Gao, X.W. Suppression of Sclerotinia sclerotiorum by the induction of systemic resistance and regulation of antioxidant pathways in tomato using fengycin produced by Bacillus amyloliquefaciens FZB42. Biomolecules 2019, 9, 613. [CrossRef]

24. Lei, S.Z.; Zhao, H.B.; Pang, B.; Qu, R.; Lian, Z.Y.; Jiang, C.M.; Shao, D.Y.; Huang, Q.S.; Jin, M.L.; Shi, J.L. Capability of iturin from Bacillus subtilis to inhibit Candida albicans in vitro and in vivo. Appl. Microbiol. Biotechnol. 2019, 103, 4377-4392. [CrossRef]

25. Liu, Y.N.; Lu, J.; Sun, J.; Zhu, X.Y.; Zhou, L.B.; Lu, Z.X.; Lu, Y.J. C16-Fengycin A affect the growth of Candida albicans by destroying its cell wall and accumulating reactive oxygen species. Appl. Microbiol. Biotechnol. 2019, 103, 8963-8975. [CrossRef]

26. Gao, S.; Wu, H.; Wang, W.; Yang, Y.; Xie, S.; Xie, Y.; Gao, X. Efficient colonization and harpins mediated enhancement in growth and biocontrol of wilt disease in tomato by Bacillus subtilis. Lett. Appl. Microbiol. 2013, 57, 526-533. [CrossRef]

27. Duitman, E.H.; Hamoen, L.W.; Rembold, M.; Venema, G.; Seitz, H.; Saenger, W.; Bernhard, F.; Reinhardt, R.; Schmidt, M.; Ullrich, C.; et al. The mycosubtilin synthetase of Bacillus subtilis ATCC6633: A multifunctional hybrid between a peptide synthetase, an amino transferase, and a fatty acid synthase. Proc. Natl. Acad. Sci. USA 1999, 96, 13294-13299. [CrossRef]

28. Leclere, V.; Bechet, M.; Adam, A.; Guez, J.S.; Wathelet, B.; Ongena, M.; Thonart, P.; Gancel, F.; Chollet-Imbert, M.; Jacques, P. Mycosubtilin overproduction by Bacillus subtilis BBG100 enhances the organism's antagonistic and biocontrol activities. Appl. Environ. Microbiol. 2005, 71, 4577-4584. [CrossRef]

29. Gu, Q.; Yang, Y.; Yuan, Q.M.; Shi, G.M.; Wu, L.M.; Lou, Z.Y.; Huo, R.; Wu, H.J.; Borriss, R.; Gao, X.W. Bacillomycin D produced by Bacillus amyloliquefaciens is involved in the antagonistic interaction with the plant-pathogenic fungus Fusarium graminearum. Appl. Environ. Microbiol. 2017, 83, e01075-17. [CrossRef]

30. Woloshuk, C.P.; Shim, W.B. Aflatoxins, fumonisins, and trichothecenes: A convergence of knowledge. FEMS Microbiol. Rev. 2013, 37, 94-109. [CrossRef]

31. Meena, K.R.; Kanwar, S.S. Lipopeptides as the Antifungal and Antibacterial Agents: Applications in Food Safety and Therapeutics. BioMed Res. Int. 2015, 2015, 473050. [CrossRef]

32. Deravel, J.; Lemiere, S.; Coutte, F.; Krier, F.; Van Hese, N.; Bechet, M.; Sourdeau, N.; Hofte, M.; Lepretre, A.; Jacques, P. Mycosubtilin and surfactin are efficient, low ecotoxicity molecules for the biocontrol of lettuce downy mildew. Appl. Microbiol. Biotechnol. 2014, 98, 6255-6264. [CrossRef]

33. Mihalache, G.; Balaes, T.; Gostin, I.; Stefan, M.; Coutte, F.; Krier, F. Lipopeptides produced by Bacillus subtilis as new biocontrol products against fusariosis in ornamental plants. Environ. Sci. Pollut. Res. 2018, 25, 29784-29793. [CrossRef]

34. Besson, F.; Peypoux, F.; Michel, G.; Delcambe, L. Antifungal activity upon Saccharomyces cerevisiae of iturin A, mycosubtilin, bacillomycin L and of their derivatives; inhibition of this antifungal activity by lipid antagonists. J. Antibiot. 1979, 32, 828-833. [CrossRef] [PubMed]

35. Sajitha, K.L.; Dev, S.A.; Florence, E.J.M. Identification and characterization of lipopeptides from Bacillus subtilis B1 against Sapstain fungus of rubberwood through MALDI-TOF-MS and RT-PCR. Curr. Microbiol. 2016, 73, 46-53. [CrossRef]

36. Han, Q.; Wu, F.L.; Wang, X.N.; Qi, H.; Shi, L.; Ren, A.; Liu, Q.H.; Zhao, M.W.; Tang, C.M. The bacterial lipopeptide iturins induce Verticillium dahliae cell death by affecting fungal signalling pathways and mediate plant defence responses involved in pathogen-associated molecular pattern-triggered immunity. Environ. Microbiol. 2015, 17, 1166-1188. [CrossRef]

37. Nasir, M.N.; Besson, F. Interactions of the antifungal mycosubtilin with ergosterol-containing interfacial monolayers. Biochim. Biophys. 2012, 1818, 1302-1308. [CrossRef] [PubMed]

38. Kim, K.; Lee, Y.; Ha, A.; Kim, J.I.; Park, A.R.; Yu, N.H.; Son, H.; Choi, G.J.; Park, H.W.; Lee, C.W.; et al. Chemosensitization of Fusarium graminearum to chemical fungicides using cyclic lipopeptides produced by Bacillus amyloliquefaciens Strain JCK-12. Front. Plant. Sci. 2017, 8, 2010. [CrossRef]

39. Hu, L.B.; Zhang, T.; Yang, Z.M.; Zhou, W.; Shi, Z.Q. Inhibition of fengycins on the production of fumonisin B-1 from Fusarium verticillioides. Lett. Appl. Microbiol. 2009, 48, 84-89. [CrossRef]

40. Gu, Q.; Zhang, C.Q.; Yu, F.W.; Yin, Y.N.; Shim, W.B.; Ma, Z.H. Protein kinase FgSch9 serves as a mediator of the target of rapamycin and high osmolarity glycerol pathways and regulates multiple stress responses and secondary metabolism in Fusarium graminearum. Environ. Microbiol. 2015, 17, 2661-2676. [CrossRef] 
41. Gu, Q.; Tahir, H.A.S.; Zhang, H.; Huang, H.; Ji, T.T.; Sun, X.; Wu, L.M.; Wu, H.J.; Gao, X.W. Involvement of FvSet1 in Fumonisin B1 biosynthesis, vegetative growth, fungal virulence, and environmental stress responses in Fusarium verticillioides. Toxins 2017, 9 , 43. [CrossRef] [PubMed]

42. Ji, F.; Xu, J.H.; Liu, X.; Yin, X.C.; Shi, J.R. Natural occurrence of deoxynivalenol and zearalenone in wheat from Jiangsu province, China. Food Chem. 2014, 157, 393-397. [CrossRef] [PubMed]

43. Plattner, R.D.; Weisleder, D.; Poling, S.M. Analytical determination of fumonisins and other metabolites produced by Fusarium moniliforme and related species on corn. In Fumonisins in Food; Jackson, L.S., DeVries, J.W., Bullerman, L.B., Eds.; Plenum Press: New York, NY, USA, 1996; Volume 392, pp. 57-64.

44. Plattner, R.D.; Desjardins, A.E.; Leslie, J.F.; Nelson, P.E. Identification and characterization of strains of Gibberella fujikuroi mating population A with rare fumonisin production phenotypes. Mycologia 1996, 88, 416-424. [CrossRef] 